\title{
Niveles de referencia para diagnóstico: Una herramienta efectiva para la protección radiológica de pacientes
}

\author{
Carlos Ubeda de la $C^{1}$, Eliseo Vaño $C^{2}$, Rafael Ruiz Cruces ${ }^{3}$, Pablo Soffia $S^{4}$, Daniella Fabri $G^{4}$.
}

1. Departamento de Tecnología Médica, Facultad de Ciencias de la Salud, Centro de Estudios en Ciencias Radiológicas, Universidad de Tarapacá, Arica, Chile.

2. Facultad de Medicina, Universidad Complutense e IdIS, Hospital San Carlos, Madrid, España.

3. Facultad de Medicina, Universidad de Málaga, Málaga, España.

4. Departamento de Imágenes, Facultad de Medicina, Clínica Alemana-Universidad del Desarrollo, Santiago, Chile.

\section{Diagnostic reference levels: An effective tool to radiological protection of patient}

Resumen: Se sabe que las exposiciones médicas con radiaciones ionizantes son actualmente la principal fuente de exposición a la radiación artificial a nivel global. Para prevenir dosis innecesariamente altas durante estas exposiciones a los pacientes, la Comisión Internacional de Protección Radiológica (ICRP) recomienda la utilización de los Niveles de Referencia para Diagnóstico (DRLs), como una herramienta efectiva de ayuda a la optimización de la protección radiológica en la exposición médica de pacientes para diagnóstico y procedimientos de intervención. Dado que la legislación chilena no tiene incorporado aún su uso, el presente trabajo de revisión tiene como objetivo, elaborar un documento guía para los profesionales de la salud y áreas afines, que contenga de manera sucinta y con recomendaciones prácticas, los principales aspectos a tener en cuenta para establecer los DRLs en procedimientos de radiodiagnóstico, intervencionismo y medicina nuclear, basados esencialmente en la publicación $N^{\circ} 135$ de la ICRP sobre DRLs.

Palabras claves: Diagnóstico por imagen, Dosis de radiación, Medicina nuclear, Nivel de referencia para diagnóstico, Protección radiológica, Radiología, Radiología intervencionista.

\begin{abstract}
It is known that medical exposition with ionizing radiation is currently the main exposition source to artificial radiation at global level. To prevent unnecessarily high doses during these patient expositions, the International Commission on Radiological Protection (ICRP) recommends the use of Diagnostic Reference Levels (DRLs), as an effective tool to support the optimization of radiological protection for patient medical exposure for diagnosis and interventional procedures. Since that the Chilean legislation does not have its use incorporated yet, the present revision work aims to elaborate a guiding document for health professionals and related fields, that succinctly and with practical recommendations, the main aspects to consider for establishing DRLs in procedures for radiodiagnostic, intervention, and nuclear medicine, mainly based in $N^{\circ} 135$ ICRP publication about DRLs.

Key words: Diagnostic reference levels, Interventional radiology, Nuclear medicine diagnostic imaging, Radiation dosage, Radiology, Radiation protection.

Ubeda $C$, et al. Niveles de referencia para diagnóstico: Una herramienta efectiva para la protección radiológica de pacientes. Rev Chil Radiol 2019; 25(1): 19-25.

${ }^{*}$ Correo electrónico: Carlos Ubeda de la Cerda / carlos.ubeda.uta@gmail.com

Trabajo enviado el 19 de diciembre de 2018. Aceptado para publicación el 05 de marzo de 2019
\end{abstract}

\section{Introducción}

El Comité Científico de Naciones Unidas sobre los Efectos de la Radiación Atómica (UNSCEAR), ha venido señalando sistemáticamente en sus últimos informes, cómo las exposiciones médicas con radia- ciones ionizantes son actualmente la principal fuente de exposición a la radiación artificial a nivel global. Esto se debe principalmente al uso cada vez mayor de procedimientos de dosis altas, en particular la tomografía computarizada (TC), pero también algunos 
procedimientos de intervencionismo y de medicina nuclear en países desarrollados (en particular los EE. UU. $)^{(1,2)}$.

Se deben adoptar medidas de protección radiológica a fin de prevenir, en lo posible, dosis innecesariamente altas durante las exposiciones médicas a los pacientes. De acuerdo a las recomendaciones de la Comisión Internacional de Protección Radiológica $(\text { ICRP) })^{(3)}$, no se recomiendan límites de dosis ni restricciones de dosis para pacientes individuales porque los mismos pueden reducir la eficacia del diagnóstico, provocando más perjuicio que beneficio. Por consiguiente, el énfasis se debe poner en la justificación de los exámenes radiológicos, en la optimización de la protección y en la utilización de los Niveles de Referencia para Diagnóstico (siendo su acrónimo en inglés, DRLs).

Los DRLs han demostrado por medio de diversos estudios, ser una herramienta efectiva que ayuda a la optimización de la protección radiológica en la exposición médica de pacientes para diagnóstico y procedimientos de intervención ${ }^{(4,5,6,7,8,9,10,11)}$. Sin embargo, su aplicación en Chile se ha visto seriamente limitada porque la legislación que regula el uso seguro de las exposiciones médicas con radiaciones ionizantes no los tiene todavía incorporados ${ }^{(12,13)}$.

Este trabajo de revisión tiene como objetivo, elaborar un documento guía para los profesionales de la salud y áreas afines, que contenga de manera sucinta y con recomendaciones prácticas, los principales aspectos a tener en cuenta para establecer los DRLs en procedimientos de radiodiagnóstico, intervencionismo y medicina nuclear, basados esencialmente en la publicación $\mathrm{N}^{\circ} 135$ de la ICRP ${ }^{(14)}$ sobre DRLs.

\section{Historia}

El concepto de DRL fue presentado por primera vez en la publicación 60 de la ICRP en el año 1990(15), donde se indicaba que: "Se debería considerar el uso de restricciones de dosis, o niveles de investigación, seleccionados por la autoridad profesional o reguladora apropiada, para su aplicación a los procedimientos diagnósticos comunes. Estas restricciones se deberían aplicar con cierta flexibilidad, con el fin de permitir el uso de ciertas dosis cuando así lo indique una fundada valoración clínica". Su uso, finalmente fue recomendado en la Publicación 73 de la misma Comisión, en el año 1996 ${ }^{(16)}$ y se indicó que: "Los niveles de referencia se usan en el diagnóstico clínico para indicar si, en condiciones de rutina, los niveles de dosis al paciente o la actividad administrada (la cantidad de material radiactivo) en un procedimiento de imagen específico, son excepcionalmente altos o bajos para ese procedimiento. En ese caso, debe comenzarse una revisión local para determinar si la protección se ha optimizado adecuadamente o si se necesita una acción correctora". En el año 2001 se publicaron algunas aclaraciones adicionales a través de una guía de apoyo de la ICRP ${ }^{(17)}$, no habiendo cambios sustanciales en los siguientes documentos de la Comisión $\left(103^{(3)}\right.$ y $\left.105^{(18)}\right)$ hasta los publicados en el año 2017, en la publicación $N^{\circ} 135^{\text {(14) }}$.

\section{Definiciones}

La ICRP ${ }^{(14)}$ recomienda el uso de cuatro términos para los DRLs:

a. DRL: Es una forma de nivel de investigación utilizada como herramienta para ayudar a la optimización de la protección en la exposición médica de pacientes sometidos a procedimientos de diagnóstico e intervencionismo.

b. Magnitud de DRL: Una magnitud que sea fácilmente medible y que permita evaluar la cantidad de radiación utilizada para realizar una tarea clínica concreta o modalidad de procedimiento. Las magnitudes y símbolos propuestos son:

a. Kerma incidente en aire $\left(\mathrm{K}_{\mathrm{a},}\right)$.

b. Kerma en aire en la superficie de entrada $\left(\mathrm{K}_{\mathrm{a}, \mathrm{e}}\right)$.

c. Kerma incidente en aire en el punto de referencia a la entrada del paciente $\left(\mathrm{K}_{\mathrm{a}, \mathrm{r}}\right)$.

d. Producto kerma en aire área $\left(P_{K A}\right)$.

e. Dosis glandular media $\left(D_{G}\right)$.

f. Producto dosis longitud (DLP).

g. Índice de dosis en tomografía computarizada (volumen) $\left(\mathrm{CTDI}_{\mathrm{vol}}\right)$.

En la tabla 1, se muestran estas magnitudes y unidades específicas para cada modalidad.

c. Valor de DRL: Un valor numérico arbitrario de una magnitud de DRL. Se habla de "valor típico de DRL" cuando se refiere a centros sanitarios que tengan varias salas de rayos $x$ o una única, o un pequeño grupo de instalaciones que utilicen una nueva técnica. En este caso se utilizada la mediana de la magnitud seleccionada. Se utiliza el percentil 75 de la distribución de las medianas de la magnitud DRL observada en unos pocos centros sanitarios (denominado "valor local de DRL") y múltiples instalaciones en todo el país (denominado "valor nacional de DRL"). Finalmente, se obtiene para múltiples países en una misma región del mundo el denominado "valor regional de DRL", a partir de los valores de la mediana de distribuciones de valores nacionales o percentil 75 de la distribución de una selección representativa de instalaciones de la región.

d. Proceso DRL: Ciclo para el establecimiento de los valores de DRLs, usándolos como una herramienta para la optimización, y luego determinar los valores actualizados de DRLs como herramientas para futuras optimizaciones. 
Tabla 1. Magnitudes para establecer niveles de referencia para diagnóstico (DRLs) por modalidad.

\begin{tabular}{|c|c|c|}
\hline Modalidad & Magnitud & Unidad \\
\hline Radiografía & $\begin{array}{l}\mathrm{K}_{\mathrm{a}, \mathrm{e}} \\
\mathrm{P}_{\mathrm{KA}}\end{array}$ & $\begin{array}{l}\text { mGy } \\
\text { mGy cm² }\end{array}$ \\
\hline $\begin{array}{l}\text { Mamografía, tomosíntesis } \\
\text { de mama }\end{array}$ & $\mathrm{K}_{\mathrm{a}, \mathrm{e},}, \mathrm{K}_{\mathrm{a}, \mathrm{i}, \mathrm{O}} \circ \mathrm{D}_{\mathrm{G}}$ & mGy \\
\hline Fluoroscopía & $\begin{array}{l}\text { PKA } \\
\mathrm{K}_{\mathrm{a}, \mathrm{r}} \\
\text { Tiempo de fluoroscopía } \\
\text { Número de imágenes en cine o } \\
\text { angiografía digital por sustracción }\end{array}$ & $\begin{array}{l}\text { Gy } \mathrm{cm}^{2} \\
\text { Gy } \\
\mathrm{s} \\
\text { Número }\end{array}$ \\
\hline $\begin{array}{l}\text { Tomografía computarizada } \\
\text { (también intervencionista) }\end{array}$ & $\begin{array}{l}\text { CTDI }_{\text {vol }} \\
\text { DLP } \\
P_{K A}\end{array}$ & $\begin{array}{l}\text { mGy } \\
\text { mGy cm } \\
\text { mGy cm² }\end{array}$ \\
\hline Medicina Nuclear & $\begin{array}{l}\text { Actividad administrada o } \\
\text { actividad por peso corporal }{ }^{\dagger}\end{array}$ & $\mathrm{MBq} \circ \mathrm{MBq} \mathrm{kg}{ }^{-1}$ \\
\hline
\end{tabular}

\section{Aspectos a considerar para establecer DRLs}

Un DRL se define para los diferentes tipos de equipos y exámenes (indicaciones clínicas) en grupos de pacientes de acuerdo a su rango de edad y fundamentalmente el peso. En general el establecimiento de DRLs se deben promover para todas las modalidades de procedimientos radiológicos, sin embargo, su valoración es baja para radiografías de extremidades, radiografías con equipos móviles (excepto cuando se trabaje en pacientes pediátricos) y densitometría ósea.

\section{a. Selección de la magnitud y valor de DRL}

Para una práctica adecuada se recomienda disponer de requisitos reglamentarios para establecer los valores de DRLs, para su aplicación y la optimización de la protección en las exposiciones médicas. En diferentes partes del mundo, la gestión de dosis a los pacientes se aborda de distintas maneras ${ }^{(19)}$. Se necesita versatilidad a la hora de establecer DRLs e implementar los programas de optimización.

Los DRLs se pueden establecer en base a la distribución de las medianas para las magnitudes de DRLs medidas en una muestra de pacientes individuales en varias áreas geográficas diferentes. Un DRL local se puede obtener a partir de un grupo (ej. 10-20) de salas de rayos $x$ o centros sanitarios. Para las áreas en las que se puedan incluir 10-20 servicios, el DRL local se establecerá como el tercer cuartil de la distribución de las medianas. Ello puede ser útil para identificar las unidades de rayos $x$ donde se requiera más atención para la optimización. Para un número menor de salas de rayos $\mathrm{x}$ o una sola instalación, se puede definir un "valor típico" como la mediana de la distribución de las dosis medidas y usarse de manera similar. En el caso de DRLs nacionales debería utilizarse una amplia muestra de centros sanitarios y de procedimientos médicos realizados con radiaciones ionizantes en ese país. Finalmente, los DRLs regionales se refieren a grupos de países en los que se hace uso de prácticas similares ${ }^{(14)}$.

\section{b. Instalaciones}

En un país desarrollado con cientos de servicios sanitarios, un muestreo muy detallado representaría una tarea muy complicada, sin embargo, una selección aleatoria de una pequeña proporción de esos centros sanitarios puede ser un buen punto de comienzo. Los resultados de $20-30$ servicios pueden ser suficientes en una primera instancia, aunque solo si se incluye un número suficiente de pacientes. En un país pequeño con menos de 50 servicios, un muestreo inicial de $30-50 \%$ de estos centros, podría bastar. En estudios subsecuentes, a medida que la infraestructura para la recopilación de datos mejora, el número de servicios incluidos se puede extender para tener una cobertura más representativa ${ }^{(14)}$.

Una vez que se han establecidos los DRLs, pueden plantearse estudios de actualización con un intervalo adecuado de 3 a 5 años, pero esto 
dependerá de las condiciones de cada país o región considerando el grado de variabilidad de los resultados de los estudios, la introducción de nuevas tecnologías o software de tratamiento de imágenes, y la disponibilidad del personal y recursos para realizar estos estudios ${ }^{(14)}$.

\section{c. Pacientes}

Como la atenuación de los rayos $\mathrm{x}$ depende de la cantidad de tejido que debe atravesar el haz de rayos $\mathrm{x}$, es importante tener en cuenta algún tipo de estándar para el tamaño del paciente. Generalmente la estandarización del tamaño del paciente se logra en base a restricciones en los intervalos de peso. Para los adultos, esto normalmente se logra al utilizar información de pacientes con un peso dentro de ciertos rangos (ej. 50-90 kg puede usarse para lograr una media de $70 \mathrm{~kg}$ ). El peso medio elegido debe acercarse al promedio de la población considerada. Para eliminar los valores atípicos y datos con errores importantes del análisis, se debe considerar algún tipo de método de exclusión (ej. eliminación del $5 \%$ de los datos ubicados en ambos extremos de la muestra). Adicionalmente, se debe tener cuidado para asegurar que los resultados no sean influenciados por una mayor cantidad de pacientes de mayor peso en algunas áreas. La variabilidad en los valores de la mediana puede ser grande en lugares donde la recopilación de datos solo sea posible para un número pequeño de pacientes. El rango intercuartil sirve como un indicador de la dispersión de los datos ${ }^{(14)}$.

Un estudio de los DRLs para un examen particular en un hospital, normalmente involucraría la recolección de información de al menos 20 pacientes para un examen radiográfico ${ }^{(20)}$, sin embargo, se requerirá los datos de más pacientes cuando haya una mayor dispersión en los resultados. Esto es especialmente más notable en la fluoroscopia, donde la diferencia en la complejidad de las patologías de los pacientes y la técnica del operador, contribuyen a la variación. Es preferible un grupo de al menos 30 pacientes dentro del rango de peso acordado para fluoroscopia diagnóstica y procedimientos de tomografía computarizada ${ }^{(20)}$. Para las mamografías, se recomienda la medición en (al menos) 50 pacientes debido a la variación en la compresión de mamas de diferente grosor. Como la variabilidad en el grosor de las mamas puede ser grande, puede ser apropiado cierta estandarización en la restricción del rango de grosor de mama incluida en el análisis ${ }^{(14)}$.

Por su parte, para establecer DRLs en pacientes pediátricos se recomienda seguir la agrupación por rangos de peso $(<5 \mathrm{~kg}, 5-<15 \mathrm{~kg}, 15-<30 \mathrm{~kg}$, $30-<50 \mathrm{~kg}$ y $50-<80 \mathrm{~kg}$ ) y edad ( $<1 \mathrm{mes}, 1 \mathrm{mes}-<4$ años, 4-<10 años, 10-<14 años y 14-<18 años) propuestos por la Comisión Europea en el año 2016 (21).

\section{d. Métodos de recolección de datos}

Existen varias opciones para la recolección de datos. Si son pocas las instalaciones, se pueden utilizar formularios impresos adaptados al examen. La llegada de los sistemas de información hospitalaria (HIS) y los sistemas de información radiológica (RIS) permiten hacer una revisión retrospectiva de la información de las pruebas de imagen a los pacientes. La recolección de datos desde el RIS tiene la ventaja de poder incluir un número mucho mayor de pacientes. El estándar de "Digital Imaging and Communication in Medicine" (DICOM) ha creado un formato específico para estos fines, denominado RDSR (informe estructurado de dosis de radiación) para el registro y almacenamiento de la información de dosis de radiación de las diferentes modalidades de imagen ${ }^{(22)}$. El sistema de gestión de dosis a los pacientes puede usar la compilación de información de los RDSRs para notificar al personal clínico y, a los físicos médicos o tecnólogos médicos de radiología responsables de la administración de estos sistemas informáticos, cuando las magnitudes dosimétricas excedan algunos de los niveles preestablecidos, especialmente cuando sean dosis altas en la piel, lo cual permite valorar un seguimiento clínico de posibles lesiones por radiación. No obstante, se necesita configurar el envío de los archivos RDSR desde las modalidades al RIS/PACS. Asimismo, por lo general, se necesita de una solución de software específico que pueda gestionar esta información.

\section{Discusión}

La medida de magnitudes relacionadas con la dosis del paciente para la optimización de la protección radiológica en imágenes médicas con radiación ionizante comenzó hace más de medio siglo. A partir de 1950, se realizaron estudios nacionales de esas magnitudes para exámenes de rayos $\mathrm{x}$ de diagnóstico en los Estados Unidos y en el Reino Unido(14). En 1970, los estudios de "Nationwide Evaluation of $x$-ray Trends" comenzaron en los Estados Unidos ${ }^{(23)}$, y en 1980, el "National Radiological Protection Board" (ahora "Public Health England")" en el Reino Unido, midieron la exposición en la superficie de entrada del paciente ${ }^{(24)}$. En el trabajo de Wall y Shrimpton ${ }^{(25)}$, se detalla el desarrollo histórico de los DRLs.

Para establecer los DRLs, la legislación nacional debe tener claramente identificadas las organizaciones responsables de cada elemento necesario para realizar esa tarea. Las organizaciones que realicen estudios a pacientes pueden ser instituciones gu- 
bernamentales, autoridades sanitarias, sociedades científicas o profesionales, instituciones académicas, hospitales, establecimientos radiológicos o clínicas. Los estudios pueden ser efectuados por tecnólogos médicos de radiología, físicos médicos u otro personal responsable de la protección radiológica.

En cuanto a las responsabilidades que se requieren para los estudios o registros de dosis a los pacientes de todo un país o región del mundo, deben ser necesariamente coordinadas por una organización con apoyo de un organismo gubernamental de cada nación. Esto requerirá de la provisión de los recursos necesarios. En algunos países, las organizaciones profesionales han creado registros continuos y diseminación de la información de las dosis de radiación a los pacientes. Como ejemplo, se puede citar Dose Index Registry del Colegio Americano de Radiología ${ }^{(26)}$.

Por otro lado, la recolección manual de la información de los datos de los pacientes puede ser engorrosa, por lo cual una buena opción para facilitar la recolección continua de datos de dosis en pacientes, puede ser preparar un registro al cual puedan enviarse esos datos. Otra opción puede ser automatizar la recolección. Puede ser posible recolectar información de un gran número de pacientes donde estén disponibles los métodos automatizados de registros de valores apropiados de magnitudes de DRLs (>100) por cada instalación ${ }^{(27,28)}$. Los registros automatizados permiten la recolección de datos de millones de exámenes ${ }^{(29)}$. Es importante destacar que el establecimiento inicial de valores de DRLs nacionales o regionales, es el primer paso en un proceso continuo de optimización.

La recolección de la información para establecer DRLs se hace a partir de procedimientos concretos (la ICRP habla de "tareas clínicas concretas") en pacientes individuales, sin embargo, hay algunas circunstancias limitadas en los que se puede evaluar la cantidad de radiación utilizada por los equipos a través de mediciones relativamente simples o con el uso de "fantomas". Estas opciones se suelen usar en radiografía dental, mamografías y, hasta cierto punto, en algunos casos de radiografías y fluoroscopia diagnóstica. Esas mediciones deberían considerarse como complementos útiles realizados durante los controles de calidad, pero, en general, aparte de la radiografía dental, no deberían reemplazar la información de los procedimientos concretos en los pacientes.

En España, actualmente se desarrolla el Proyecto DOPOES 2 (2017-2020), entre el Consejo de Seguridad Nuclear y la Universidad de Málaga para la realización de un estudio sobre aplicación de niveles de referencia de dosis (DRLs) en los procedimientos de radiodiagnóstico médico en pacientes, utilizados en los centros sanitarios españoles, así como su contribución a las dosis recibidas por la población. Su objetivo es determinar los DRLs a partir de las dosis impartidas a pacientes, adultos y pediátricos, basándose en las magnitudes propuestas en los estudios europeos ${ }^{(30)}$.

Los DRLs cobran especial importancia en TC, ya que como se ha señalado, esta modalidad es la principal fuente artificial de radiación a la que está expuesta la población y además, es una técnica de mayor complejidad, con gran variabilidad en los equipos y en las opciones de operación ${ }^{(31)}$. En TC, los DRLs se deben estudiar en base a los diferentes protocolos de examen, es decir al conjunto de parámetros o características ajustadas a procedimientos clínicos concretos y a un área anatómica específica, por ejemplo, abdomen y pelvis sin y con contraste intravenoso. Así, habrá un DRL para cada protocolo de TC, basado en los datos de un número determinado de pacientes (cuanto más cercano al total, será más representativo). En el caso de las TC pediátricas, además de la segmentación por protocolo, habrá que hacerlo por edad y/o peso. En este sentido, es muy importante que los protocolos estén correctamente definidos y ordenados, de manera que la terminología sea coincidente con la realidad. Por ejemplo, si se usa un protocolo de tórax para hacer un abdomen, el DRL no será totalmente representativo. Recientemente, la Sociedad Radiológica de Norteamérica (RSNA) ha publicado un catálogo de términos para estandarizar el nombre de los protocolos (Radlex), a fin de facilitar la comparación de DRL entre diferentes centros y/o países ${ }^{(32)}$. Si bien los DRLs pueden calcularse manualmente en base a recolección discreta de datos en grupos de pacientes, en la actualidad existen soluciones informáticas específicas para registro, administración y gestión de dosis de radiación (radiation tracking software $)^{(33)}$. Estas herramientas de software permiten establecer un flujo de información entre las modalidades (por ejemplo, TC) y el PACS, enviando los archivos RDSR, así como imágenes referenciales y parámetros de adquisición, lo que posteriormente son almacenados en un servidor y analizados en una plataforma gráfica basada en un servidor web. Gracias a la difusión de estos sistemas, hoy es posible automatizar la elaboración de DRLs en base a grandes cantidades de pacientes ${ }^{(34)}$.

\section{Agradecimientos}

El investigador Carlos Ubeda agradece el apoyo de la Dirección de Investigación de la Universidad de Tarapacá, a través del proyecto de investigación UTA Mayor № 7713-18.

\section{Referencias}

1. UNSCEAR. Sources and Effects of lonizing Radiation. Volume I: Sources. UNSCEAR 2000 Report. United Nations Scientific Committee on the Effects of Atomic Radiation, 2000 Report to the General Assembly, with scientific annexes. United Nations sales publication E.00.IX.3. United Nations, New York, 2000: 1-659. 
2. UNSCEAR. Sources and Effects of lonizing Radiation. Volume I: Sources: Report to the General Assembly, Scientific Annexes A and B. UNSCEAR 2008 Report. United Nations Scientific Committee on the Effects of Atomic Radiation. United Nations sales publication E.10.XI.3. United Nations, New York, 2010: 1-106.

3. International Commission on Radiation Protection (ICRP). The 2007 Recommendations of the International Commission on Radiological Protection. ICRP 103. Ann ICRP 2007; 37: 2-4.

4. Jensen, J.E., Butler, P.F. Breast exposure: nationwide trends; a mammographic quality assurance program - results to date. Radiol Technol 1978; 50: 251-257.

5. Hart, D., Hillier, M.C., Wall, B.F. National reference doses for common radiographic, fluoroscopic and dental X-ray examinations in the UK. Br. J. Radiol 2009; 82: 1-12.

6. Hart, D., Hillier, M.C., Shrimpton, P.C. Doses to Patients from Radiographic and Fluoroscopic X-ray Imaging Procedures in the UK-2010 Review Report. HPA-CRCE-034. Health Protection Agency, Chilton. 2012. Disponible en: https://www.gov.uk/government/ publications/diagnostic-radiology-national-diagnosticreference-levels-ndrls. (Accesado el 28/Jun/2018).

7. Hesse, B., Tagil, K., Cuocolo, A., et al. EANM/ESC procedural guidelines for myocardial perfusion imaging in nuclear cardiology. Eur. J. Nucl. Med. Mol. Imag. 2005; 32: 855-897.

8. Padovani, R., Vaño, E., Trianni, A., et al.. Reference levels at European level for cardiac interventional procedures. Radiat. Prot. Dosim. 2008; 129: 104-107.

9. Botros, G.M., Smart, R.C., Towson, J.E. Diagnostic reference activities for nuclear medicine procedures in Australia and New Zealand derived from the 2008 survey. ANZ Nucl. Med. 2009; 40: 2-11.

10. Miller, D.L., Kwon, D., Bonavia, G.H. Reference levels for patient radiation doses in interventional radiology: proposed initial values for U.S. practice. Radiology. 2009; 253: 753-764.

11. Miller, D.L., Hilohi, C.M., Spelic, D.C. Patient radiation doses in interventional cardiology in the U.S.: advisory data sets and possible initial values for U.S. reference levels. Med. Phys. 2012; 39: 6276-6286.

12. Reglamento de protección radiológica de instalaciones radiactivas, Decreto Supremo №3 de la República de Chile, 3 de Enero de 1985.

13. Reglamento sobre autorizaciones para instalaciones radioactivas o equipos generadores de radiaciones ionizantes, personal que se desempeña en ellas, $u$ opere tales equipos y otras actividades afines, Decreto Supremo №133 de la República de Chile, 22 de mayo de 1984.

14. International Commission on Radiation Protection (ICRP). The 2017 Diagnostic reference levels in medical imaging. ICRP Publication 135. Ann. ICRP 2017; 46(1): 1-143.

15. International Commission on Radiation Protection (ICRP). 1990 Recommendations of the International Commission on Radiological Protection. Publication 60. Ann. ICRP 1991; 21(1-3):1-201.

16. International Commission on Radiation Protection (ICRP). Radiological protection and safety in medicine. ICRP Publication 73. Ann. ICRP 1996; 26(2): 1-47.

17. International Commission on Radiation Protection
(ICRP). Diagnostic reference levels in medical imaging: review and additional advice. ICRP Supporting Guidance 2. Ann. ICRP 2001; 31(4): 1-14. Disponible en: http://www.icrp.org/docs/DRL_for_web.pdf. (Accesado el 12/Feb/2018).

18. International Commission on Radiation Protection (ICRP). Radiation protection in medicine. ICRP Publication 105. Ann ICRP 2007; 37(6): 1-63.

19. Martin, C.J., Le Heron, J., Borras, C., Sookpeng, S., Ramirez, G., 2013. Approaches to aspects of optimisation of protection in diagnostic radiology in six continents. J. Radiol Prot 2013; 33: 711-734.

20. Disponible en: https://assets.publishing.service.gov.uk/ government/uploads/system/uploads/attachment_data/ file/337175/National_Protocol_for_Patient_Dose_Measurements_in_Diagnostic_Radiology_for_website. pdf. (Accesado el 08/Feb/2019)

21. European Comission (EC). European Guidelines on Diagnostic Reference Levels for Paediatric Imaging. Radiation Protection 185. European Union, Luxembourg, 2016.

22. DICOM Standards Committee. DICOM standard supplement 127: CT radiation dose reporting. Rosslyn, Va: National Electrical Manufacturers Association, 2007.

23. FDA. Nationwide Evaluation of X-ray Trends (NEXT) Eight Years of Data (1974-1981). US Department of Health and Human Services, Public Health Service, Food and Drug Administration, Silver Spring, MD, 1984.

24. Shrimpton, P. C., Wall, B. F., Jones, D. G., Fisher, E. S., Hillier, M. C., Kendall, G. M. and Harrison, R. M. A National Survey of Doses to Patients Undergoing a Selection of Routine X-ray Examinations in English Hospitals. NRPB-R200 (London: HMSO), 1986.

25. Wall, B. F and Shrimpton, P. C. The historical development of reference doses in diagnostic radiology. Radiation Protection Dosimetry 1998; 80: 15-20.

26. Disponible en: https://www.acr.org/Practice-Management-Quality-Informatics/Registries/Dose-IndexRegistry._(Accesado el 08/Feb/2019)

27. Goenka, A.H., Dong, F., Wildman, B., et al. CT radiation dose optimization and tracking program at a large quaternary-care health care system. J. Am Coll Radiol 2015; 12: 703-710.

28. MacGregor, K., Li, I., Dowdell, T., Gray, B.G. Identifying institutional diagnostic reference levels for CT with radiation dose monitoring software. Radiology 2015; 276: 507-517.

29. Bhargavan-Chatfield, M., Morin, R.L., 2013. The ACR computed tomography dose index registry: The 5 million examination update. J. Am Coll Radiol 2013; 10: $980-983$.

30. Ruiz-Cruces, R, Cañete, S, Perez-Martinez, M. Proyecto DOPOES 2. Boletín Oficial Estado Español (BOE). 2018, 66 Sección III. 30733-30753.

31. Parakh A, Kortesniemi M, Schindera ST. CT Radiation Dose Management: A Comprehensive Optimization Process for Improving Patient Safety. Radiology 2016; 280: 663-73.

32. Vreeman DJ, Abhyankar S, Wang KC, Carr C, Collins $\mathrm{B}$, Rubin DL, Langlotz CP. The LOINC RSNA radiology playbook - a unified terminology for radiology procedures, J Am Med Inform Assoc. Julio 1 de 2018; 
25(7): 885-893. doi: 10.1093/jamia/ocy053.

33. Cook TS, Zimmerman S, Maidment AD, Kim W, Boonn WW. Automated extraction of radiation dose information for CT examinations. J Am Coll Radiol
2010; 7(11): 871-877.

34. Wilson JM, Samei E. Implementation of the ACR Dose Index Registry. J Am Coll Radiol 2015; 12(3): 312-313. 